\title{
Endocarditis Due to Vancomycin-Resistant Enterococccus gallinarum in a Patient with End-Stage Renal Failure: A Case Report
}

\author{
Gulden Ersoz $^{1 *}$, Mustafa Uguz ${ }^{1}$, Barlas N. Aytacoglu², Ali Kaya ${ }^{1}$ \\ ${ }^{1}$ Department of Infectious Diseases and Clinical Microbiology, Faculty of Medicine, Mersin University, Mersin, Turkey, ${ }^{2}$ Department \\ of Cardiovascular Surgery, Faculty of Medicine, Mersin University, Mersin, Turkey. \\ Email: "gersoz@mersin.edu.tr
}

Received December $13^{\text {th }}$, 2011; revised January $15^{\text {th }}$, 2012; accepted January $31^{\text {st }}, 2012$

\begin{abstract}
The first described vancomycin resistant enterococci (VRE) was about twenty years ago. Recently VRE have been reported by many clinics. However endocarditis due to VRE is still a rare entity and there are only a few cases reported in the literature. We are reporting a 59-year-old male patient with chronic renal failure who was on hemodialysis. He presented with a sudden onset of fever, tachycardia and respiratory distress. The performed echocardiography revealed vegetations on the mitral and aortic valves. As he was diagnosed to have infective endocarditis the patient was put on ampicillin and gentamicin therapy. He underwent an emergent mitral and aortic valve surgery due to ensued heart failure. While he was still on ampicillin and gentamicin therapy, E. gallinarum, which was resistant to vancomycin (MIC = $8 \mathrm{mg} / \mathrm{L}$ ), was isolated from the surgical valve specimens and hence his antibiotic regime was switched to teicoplanin (MIC $<0.5 \mathrm{mg} / \mathrm{L}$ ). 28 days after teicoplanin therapy the patient was discharged with free of symptoms and any complication. This patient is presented as an example for an endocarditis with an unusual type of enterococci.
\end{abstract}

Keywords: Infective Endocarditis; Vancomycin-Resistant Enterococci; E. gallinarum

\section{Introduction}

For the last couple of years the enterococci that are a member of the gastrointestinal (GI) flora have begun to become more of an issue among hospital infections as an important gram positive infection in the form of vancomycin resistant enterococci (VRE) [1]. The responsible factors for the vancomycin resistance are usually the VanA and VanB genes that are inheritable. We usually come across with the E. faecalis $(80 \%-90 \%)$ and $E$. faecium $(10 \%-15 \%)$ as the VRE. The VanC that causes a low level of vancomycin resistance gives rise to an intrinsic resistance, which is specific for E. gallinarum (VanC-1) and E. casseliflavus/flavescens (VanC-2) types [2]. The clinical importance of these phenotypes is less when compared with other VREs however bacteriemia with these phenotypes are reported to be more common in cases that have diabetes or solid or hematological malignancies. Enterococci as the causative agent of endocarditis were reported to be mainly in the diabetic patients. In this report we are forwarding a diabetic case, which was under chronic hemodialysis and presented with infective endocarditis (IE) due to E. gallinarum that

\footnotetext{
*Corresponding author.
}

was resistant to vancomycin.

\section{Case Presentation}

A 59-year-old male patient who was on regular hemodialysis program for terminal stage renal failure applied with high fever, shortness of breath and palpitation following a hemodialysis application. His history revealed that he had non insulin dependent type II diabetes mellitus for the last 12 years, had a coronary arterial stent placed 2 years ago and he complained of having intermittent fever during the last 30 days. The patient had a permanent dialysis catheter placed for the last 11 months and having being diagnosed to have a catheter infection caused by methicilline sensitive staphylococcus aureus (MSSA) 3 months ago he received an ampicillin/sulbactam therapy for 15 days. His catheter was not renewed. He underwent hemodialysis at the same center and did not receive a lock solution containing an antibiotic at the termination of hemodialysis.

In his physical examination his arterial blood pressure was 120/80 mmHg, his body temperature was $38.6^{\circ} \mathrm{C}$ and pulse rate was $130 \mathrm{~b} / \mathrm{m}$. Cardiac examination revealed $3 / 6$ systolic and diastolic murmurs at the mitral and aortic auscultation areas over the chest. He underwent a trans- 
thoracic echocardiography (TTE), which showed severe aortic and mitral insufficiency and vegetations on both valves that were leading to turbulent blood flow. The laboratory tests put forward an increase in the leukocyte count $\left(18,740 / \mathrm{mm}^{3}\right)$, low hemoglobin level $(8.6 \mathrm{~g} / \mathrm{dL})$, an increased erythrocyte sedimentation rate (ESR) of 111 $\mathrm{mm} / \mathrm{h}$, a thrombocyte count of $228.000 / \mathrm{mm}^{3}$ and a C reactive protein level of $90 \mathrm{mg} / \mathrm{L}$ (normal value: $<5$ $\mathrm{mg} / \mathrm{L}$ ). The urea in blood was found to be $100 \mathrm{mg} / \mathrm{dL}$ (normal value is $13-15 \mathrm{mg} / \mathrm{dL}$ ), creatinine was 5.5 $\mathrm{mg} / \mathrm{dL}$ (normal value is $<1.2 \mathrm{mg} / \mathrm{dL}$ ), alanine transaminase (ALT) was $68 \mathrm{U} / \mathrm{L}$, apartate transaminase (AST) was $84 \mathrm{U} / \mathrm{L}$ and albumin was $3.4 \mathrm{~g} / \mathrm{dL}$.

Keeping in mind that the patient had a permanent catheter for hemodialysis and with the presence of fever and echocardiograhic findings of the vegetations leading to turbulent blood flow the patient was diagnosed to have infective endocarditis and he was hospitalized. After obtaining peripheral blood and urine samples for culturing for the discrimination of the responsible microorganism, he was put on an antibiotic therapy, which consisted of ampicillin $12 \mathrm{~g} / \mathrm{d}$ and gentamicin $160 \mathrm{mg} / \mathrm{d}$. His fever subsided following the antibiotic regimen however his cardiac failure persisted. The laboratory findings on the third day of therapy were as follows: CRP: $103 \mathrm{mg} / \mathrm{dL}$, Urea: $195 \mathrm{mg} / \mathrm{dL}$, creatinine: $6.7 \mathrm{mg} / \mathrm{dL}$, ALT: $190 \mathrm{U} / \mathrm{L}$, AST: $81 \mathrm{U} / \mathrm{dL}$ and Albumin: $2.9 \mathrm{~g} / \mathrm{dL}$. The patient underwent a transoesophageal echocardiographic (TEE) evaluation the results of which supported the findings of the previous TTE. The leaflets were found to be perforated, the vegetations were observed to be mobile and the valve was regurgitating thus he was transferred to the Department of Cardiovascular Surgery for an emergency operation. The patient underwent a mitral valve replacement (MVR) and aortic valve replacement (AVR) and the explanted valve specimens were sent for culture (Figure 1). He continued to receive the same antibiotic regime postoperatively until on the $4^{\text {th }}$ postoperative day when the results of the culture from the valve specimens indicated to vancomycin resistant $E$. gallinarum (vancomycin MIC $=8 \mathrm{mg} / \mathrm{L}$ ) [2]. The continuation of the elevation of the laboratory parameters in CRP, ALT, urea and creatinine and the result of the culture from the explanted valve specimens led us to terminate the ongoing therapy and we replaced the antibiotic regime with teicoplanin [MIC $<0.5 \mathrm{mg} / \mathrm{L}$, E test strip, (AB Biodisk, Solna, Sweden)] $800 \mathrm{mg} / \mathrm{d}$ (the first 3 doses were given in 3 hours intervals). The patient received this therapy for 28 days. He was discharged from the hospital by the end of this therapy with no clinical or laboratory complications.

\section{Discussion}

In this report we presented a case, which had permanent hemodialysis catheter for the last 11 months and presented with infective endocarditis with the involvement of two cardiac valves. The responsible agents, the enterococci, that lead to severe clinical conditions, have gained importance in terms of glycopeptide resistance and nosocomial infections during the recent years [1]. In his report that evaluated 2212 IE patients McDonald et al. denoted that the streptococci were the mostly encountered responsible agent of IE however they also stated that enterococci were also isolated in $4.8 \%$ of the cases [3]. E. faecalis were $58 \%$ of these strains. The streptococci that are the most commonly encountered infecting agents give rise to infection mostly in patients with no underlying diseases however enterococci are more frequently isolated in patients who usually have permanent catheters, diabetes or malignancies [3]. Despite the vancomycin resistance ratios have not been given in their manuscript [3], the frequency of infections with vancomycin resistant enterococci have been found out to be widely increasing in infections related with health care services. The presence of resistant microorganisms emerges as a risk factor for treatment failure.

E. gallinarum is a more rarely encountered infecting agent when compared with E. faecalis and E. faecium. Important risk factors for the development of such infections consist of gastrointestinal interventions and biliary drainage. When the literature in English language was reviewed only 3 cases of infective endocarditis with $E$. gallinarum were reported in a series of 20 bacteremia cases [4-6]. The underlying diseases in each of the three patients have identified to be uretero-ileostomy in one, history of being undergone a gastrointestinal and biliary tract surgery in the other and diabetes mellitus and coronary artery stent placement in the third patient. In the series of Reid et al. there was a history of antibiotic usage within the last 3 months [9]. Vancomycin resistant $E$. gallinarum is particularly seen to be the cause of infection

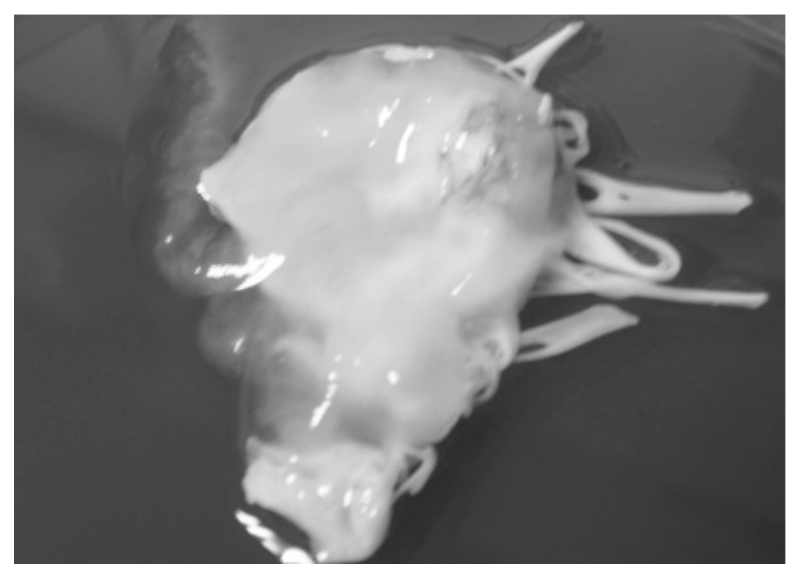

Figure 1. Leaflets that were explanted during valvectomy which were also used as specimens for culture. 
in patients that have undergone gastrointestinal intervention with a history of being using antibiotics. Our patient had a history of having cardiac intervention with subsequent use of antibiotics.

It is clearly well known that the presence of a permanent catheter is an important risk factor for the development of endocarditis. The commonly encountered causative agent in such patients is $S$. aureus. Spies et al. studied 40 infective endocarditis cases with terminal stage renal failure and found out S.aureus as the responsible microorganism in $50 \%$ of the patients [7]. Still, in the second line it was the enterococci, which formed $23 \%$ of the infections [7]. In 53\% of the cases only the mitral valves were involved and in $20 \%$ both aortic and mitral valves were affected. Both valves in our patient who had a permanent catheter were involved. Such patients should always be cautiously evaluated and followed up in terms of infection on the second valve.

In our case cardiac failure ensued due to floating vegetations on both valves and perforation on the mitral valve with no other complications like embolic events and hence the patient underwent an emergency valve replacement. Endocarditis with $S$. aureus usually proceeds with embolic complications however enterococcal infections present with less complications and are usually less severe in terms of clinical presentation [8]. Surgical indications for native valve endocarditis account for approximately $40 \%-50 \%$ of the cases [8]. Although the presence of coagulase negative staphylococcus and abscess formation especially in the young generation give rise to a more frequent determination for a surgical intervention, the deterioration of the hemodynamic status still constitutes the first line determinant for surgery. Although no compared studies have been performed in cases with severe valve insufficiencies where mortality rates are high, studies where the outcome of surgery was compared to medical therapy did not put forward the superiority of surgery to medical therapy in terms of mortality. Nevertheless, surgery is recommended in cases with ensuing cardiac failure and abscess formation in order to provide better tissue penetration of the antibiotics [8-10].

In our case E. gallinarum was isolated with the phenotype VanC, low level of vancomycin resistance, and teicoplanin sensitivity $[2,11]$. Moreover there was a high level of gentamicin resistance as well. E. casseliflavus/ flavescens and E. gallinarum with VanC phenotypic properties are observed less than $5 \%$. In a Japanese study, which evaluated enterococcal bacteremiae for 6 years, VanC type enterococci were found to be responsible from $12 \%(n=9)$ of them [12]. For all the strains MIC values for vancomycin were $4 \mathrm{mg} / \mathrm{L}$ and they were sensitive to teicoplanin, ampicillin and linezolid. All the patients in this series were treated with fourth generation cephalosporins or carbapenems. Despite ampicillin and penicilline are effective and recommended treatment agents in the literature in these patients the majority of whom had malignancies as the underlying main problem with clinically bad conditions during admission together with the isolation of different microorganisms apart from enterococci, no change in the treatment protocols was accomplished. Ampicillin and gentamicin were the first antibiotics commenced for the treatment of our patient once he received the diagnosis of having infective endocarditis however he was put teicoplanin treatment on following the growth of enterococcus from the vegetations of the surgically explanted valvular specimens. The identification of the infecting agent as E. gallinarum and discovery of its sensitivity to teicoplanin guided us to carry on his antibiotic regime with this antibiotic. The regimen continued for 4 weeks and terminated by the end of this period with the patient being well clinically as well.

As a conclusion, it should always be kept in mind that in patients with terminal stage renal failure in whom permanent dialysis catheters have been placed infections with resistant microorganisms that have lower incidence and different from routinely encountered ones might ensue. The importance of enterococcal infections is being increased day by day especially in terms of nosocomial infections. This case who experienced infective endocarditis due to E. gallinarum with VanC phenotypic resistance may be a guide in the evaluation of similar cases.

\section{REFERENCES}

[1] W. C. Huskins, M. C. Huckabee, P. N. O’Grady, P. Murray, H. Kopetskie, L. Zimmer, M. E. Walker, R. L. Sinkowitz-Cochran, J. A. Jernigan, M. Samore, D. Wallace and D. A. Goldmann, "Investigators Intervention to Reduce Transmission of Resistant Bacteria in Intensive Care,” New England Journal of Medicine, Vol. 364, No. 15, 2011, pp. 1407-1418. doi:10.1056/NEJMoa1000373

[2] K. Dogson, "VRE Detection: A New Gold Standard," Clinical Microbiology Newsletter, Vol. 26 No. 4, 2004, pp. 25-30. doi:10.1016/S0196-4399(04)90013-X

[3] R. McDonald, L. Olaison, D. J. Anderson, B. Hoen, J. M. Miro, S. Eykyn, E. Abrutyn, V. G. Fowler Jr., G. Habib, C. Selton-Suty, P. A. Pappas, C. H. Cabell, G. R. Corey, F. Marco and D. J. Sexton "Enterococcal Endocarditis: 107 Cases from the International Collaboration on Endocarditis Merged Database” American Journal of Medicine, Vol. 118, No. 7, 2005, pp. 759-766. doi:10.1016/j.amjmed.2005.02.020

[4] K. C. Reid, III F. R. Cockerill and R. Patel "Clinical and Epidemiological Features of Enterococcus casseliflavus/ flavescens and Enterococcus gallinarum Bacteremia: A Report of 20 Cases” Clinical Infectious Diseases, Vol. 32, No. 11, 2001, pp. 1540-1546. 
[5] S. Dargere, M. Vergnaud, R. Verdon, E. Saloux, O. Le Page, R. Leclercq and C. Bazin, "Enterococcus gallinarum Endocarditis Occurring on Native Heart Valves" Journal of Clinical Microbiology, Vol. 40, No. 6, 2002, pp. 2308-2310. doi:10.1128/JCM.40.6.2308-2310.2002

[6] M. Ortu, E. Gabrielli, I. Caramma, R. Rossotti, M. Gambirasio and C. Gervasoni, "Enterococcus gallinarum Endocarditis in a dIabetic Patient," Diabetes Research and Clinical Practice, Vol. 81, No. 1, 2008, pp. e18-20. doi:10.1016/j.diabres.2008.03.016

[7] C. Spies, J. R. Madison and I. J. Schatz "Infective Endocarditis in Patients with End-Stage Renal Disease: Clinical Presentation and Outcome," Archives of Internal Medicine, Vol. 164, No. 1, 2004, pp. 71-75. doi:10.1001/archinte.164.1.71

[8] J. R. McDonald “Acute Infective Endocarditis,” Infectious Diseases Clinical of North American, Vol. 23, No. 3, 2009, pp. 643-664. doi:10.1016/j.idc.2009.04.013

[9] H. R. Vikram, J. Buenconsejo, R. Hasbun and V. J. Quagliarello "Impact of Valve Surgery on 6-Month Mortality in Adults with Complicated, Left-Sided Native Valve Endocarditis” JAMA, Vol. 290, No. 24, 2003, pp. 3207-3214. doi:10.1001/jama.290.24.3207

[10] D. J. Sexton and D. Spelman "Current Best Practices and Guidelines: Assessment and Management of Complications in Infective Endocarditis," Cardiology Clinics, Vol. 21, No. 2, 2003, pp. 273-282. doi:10.1016/S0733-8651(03)00031-6

[11] S. H. Choi, S. O. Lee, T. H. Kim, J. W. Chung, E. J. Choo, Y. G. Kwak, M. N. Kim, Y. S. Kim, J. H. Woo, J. Ryu and N. J. Kim "Clinical Features and Outcomes of Bacteremia Caused by Enterococcus casseliflavus and Enterococcus gallinarum: Analysis of 56 Cases,” Clinical Infectious Diseases, Vol. 38, No. 1, 2004, pp. 53-61. doi:10.1086/380452

[12] H. Koganemaru and S. Hitomi "Bacteremia Caused by VanC-Type Enterococci in a University Hospital in Japan: A 6-Year Survey," Journal of Infection and Chemotherapy, Vol. 14, No. 6, 2008, pp. 413-417. doi:10.1007/s10156-008-0644-X 